\title{
Modélisation numérique des avalanches aérosols
}

\author{
M. Naaim \\ Division Nivologie du Cemagref
}

\section{IINTRODUCTION}

On appelle courant de turbidité les écoulements dans lesquels un fluide lourd, constitué soit par une solution concentrée de type solution saline, soit par une suspension de particules (grains de sables, poussières, particules de neige), s'écoule dans un fluide de moindre densité, sous l'effet de la gravité. Cette différence de densité entre les deux fluides est le moteur de ce type d'écoulement. Ces phénomènes sont souvent décrits par le terme d'écoulement de densité ou d'écoulement turbidique. Les exemples de tels écoulements sont nombreux : masse de gaz lourd s'écoulant à la surface du sol, courants turbidiques sousmarins, écoulements pyroclastiques libérés lors des éruptions volcaniques (nuées ardentes) et avalanches aérosols souvent appelées avalanches poudreuses.

Les avalanches poudreuses s'observent généralement en plein hiver sous nos latitudes, ou en très haute montagne comme sur l'Himalaya ou sur les Andes, après de fortes chutes de neige sèche, légère et sans cohésion. Quand l'épaisseur de la couche de neige fraîche atteint 30 à $60 \mathrm{~cm}$, il suffit d'une faible contrainte pour ébranler le manteau neigeux trop instable, et provoquer une avalanche.

Quand la masse de neige glisse sur une pente, sa vitesse augmente sous l'effet de la gravité. Il se forme alors un cisaillement important entre la masse glissante et l'air. Lorsque la vitesse devient suffisante, une sorte d'érosion du matériau dense s'amorce en partie frontale de l'écoulement. La contrainte de cisaillement devient supérieure à la contrainte interne du matériau. Des particules sont alors arrachées à l'écoulement dense et mises en suspension dans une zone où règne une intense turbulence. Le volume de l'aérosol ainsi formé grandit en incorporant de l'air dans sa partie supérieure et des particules de neige dans sa partie basale.

En présence de forte pente, la vitesse de l'avalanche augmente ainsi que son pouvoir érosif. Parallèlement à l'incorporation de la neige, l'avalanche incorpore une quantité non négligeable d'air ambiant. L'existence simultanée de ces deux phénomènes donne lieu à des avalanches extrêmement dangereuses. Quand la pente devient faible, le terme moteur (gravité) devient faible. Les effets dissipatifs turbulents ou visqueux et la sédimentation deviennent prépondérants et finissent par arrêter l'avalanche.

\section{MODÉLISATION NUMÉRIQUE DES AVALANCHES AÉROSOLS}

\subsection{Variables et hypothèses}

Les écoulements naturels de type avalanche aérosol peuvent être considérés comme des écoulements diphasiques.
Il s'agit de l'écoulement d'un fluide lourd formé d'air et de particules de neige en suspension dans un fluide léger constitué de l'air seul. La différence de densité engendre l'écoulement grâce à l'effet de la gravité. L'écoulement est donc étudié comme un écoulement gravitaire de type fluide lourd dans un fluide léger.

La modélisation numérique proposée est basée sur les équations de Navier Stokes moyennant les hypothèses suivantes :

- L'air est considéré comme un gaz parfait à comportement newtonien. On considère que les particules en suspension ne modifient pas la loi de comportement.

- On considère que ses particules en suspension suivent le mouvement du gaz avec un glissement vertical dû à la vitesse de chute des particules dans l'air.

On se place dans un repère galiléen formé de trois axes : l'axe $x$ et l'axe $y$ forment le plan horizontal et l'axe $z$ est la verticale ascendante. Dans ce repère, le vecteur gravité est donné par $\vec{g}=(0,0,-g)^{t}$. Les variables sont : $\rho_{g}$ la masse volumique de l'air, $\rho_{s}$ est la masse volumique de la neige, $\vec{u}=(u, v, w)^{t}$ le vecteur vitesse et $p$ la pression.

On considère la concentration volumique en particules solides, définie comme le rapport entre le volume occupé par ces particules et le volume total : $c=v_{s} /\left(v_{g}+v_{\mathrm{s}}\right)$ où $v_{s}$ est le volume occupé par les particules en suspension et $v_{g}$ est le volume occupé par l'air. Basée sur ces considérations, la densité moyenne est liée à la densité des particules solides et à la densité du gaz ambiant par la relation : $\rho_{m}=\rho_{g}+c \rho_{s}$.

\subsection{Equations de base}

Les équations' régissant l'écoulement sont :

i) Conservation de la masse d'air :

$$
\frac{\partial \rho_{g}}{\partial t}+\operatorname{div}\left(\rho_{g} \vec{u}\right)=0
$$

ii) Conservation de la masse des particules

$$
\frac{\partial c}{\partial t}+\operatorname{div}(c \vec{u})=-\frac{\partial c w_{g}}{\partial z}+k_{c} \Delta c
$$

$k_{c}$ est la diffusion moléculaire de la concentration.

$w_{g}$ est la vitesse de chute des particules solides dans l'air. 
iii) Conservation de la quantité de mouvement:

$$
\frac{\partial \rho \vec{u}}{\partial t}+\operatorname{div} \vec{F}+\vec{\nabla}(P)=c \rho_{s} g+\mu \Delta(\vec{u})
$$

où

$$
\vec{F}=[u \cdot(\rho \vec{u}) v \cdot(\rho \vec{u}) w \cdot(\rho \vec{u})]^{t}
$$

- On considère que l'influence des particules sur la pression du gaz est négligeable. La pression de l'air se détermine à partir de l'équation d'état suivante :

$$
p=k \rho_{g}^{\gamma} \quad \text { avec } \quad \gamma>1
$$

La célérité du son s'écrit alors :

$$
c=\sqrt{k} \rho \frac{\gamma-1}{2}
$$

\subsection{Modèles de turbulence}

Les équations d'évolution des diverses grandeurs physiques étant déterminées, il s'agit maintenant de réécrire ces équations en prenant en compte les effets de la turbulence. La méthode consiste à paramétrer toutes les échelles de la turbulence en décomposant les grandeurs physiques en quantités moyennes et fluctuantes : c'est la décomposition de Reynolds. Elle permet de prendre en compte les effets dissipatifs dus aux petites structures de la turbulence, en modélisant seulement l'écoulement moyen. Cela revient à décomposer les variables instantanées en une partie moyenne et une partie fluctuante. En effet, seule la connaissance des grandeurs moyennes est intéressante dans les écoulements étudiés. Il faut alors procéder à un traitement statistique des équations avant toute résolution numérique. L'approche statistique appliquée à un processus non linéaire conduit toujours à un accroissement du nombre d'inconnues, ce qui pose le problème de fermeture. D'autre part, pour permettre une confrontation expérimentale, on assimile les moyennes statistiques aux moyennes temporelles, même si dans le cas présent, l'écoulement est fortement instationnaire.

Le traitement statistique permet d'aboutir aux équations moyennes du mouvement, mais on note l'apparition de corrélations d'ordre 2. Cela provient de la non-linéarité des équations de Navier Stokes. Le fait de les transformer en contraintes turbulentes et de les regrouper aux autres contraintes moyennes existantes, préconditionne déjà la forme de modélisation. L'idée est d'emprunter la même démarche que celle adoptée lors de la formulation de la loi de comportement newtonienne. On obtient alors la loi dite de Boussinesq (appelée aussi loi de viscosité tourbillonnaire), appartenant à la classe des modèles au concept de viscosité turbulente.

La loi de Boussinesq fait apparaître la viscosité turbulente qu'il faut maintenant expliciter pour fermer le système. Mais cette viscosité doit être caractérisée par des échelles représentatives du mouvement turbulent luimême. Elle est donc fortement liée au mouvement moyen, et apparaît donc comme une propriété de l'écoulement. Elle n'est pas constante dans le domaine d'écoulement car les échelles de longueur et de vitesse dont dépend la viscosité turbulente, ne sont pas indépendantes du mouvement macroscopique moyen et sont évolutives dans l'espace et dans le temps. Une première approche de schéma de fermeture est le schéma de fermeture de type longueur de mélange. C'est le modèle à zéro équation. Mais ce modèle n'est pas suffisamment universel, du fait qu'il suppose une connaissance préalable de l'écoulement, de la loi d'évolution de la longueur de mélange et de la viscosité turbulente. Le modèle à une équation permet de relier l'échelle de vitesse caractéristique du mouvement fluctuant à l'énergie cinétique de la turbulence. L'équation exacte de transport de l'énergie cinétique de la turbulence, directement déduite des équations de Navier Stokes, permet de calculer cette énergie turbulente. Cependant de nouvelles corrélations inconnues apparaissent, et le problème de fermeture se pose à nouveau. La modélisation de cette équation se base alors sur les mécanismes physiques de la cascade énergétique de Kolmogorov dans le cadre d'écoulements franchement turbulents. L'échelle de la longueur de mélange est là aussi donnée empiriquement. Le dernier modèle de ce type est le modèle à deux équations. Il permet de s'affranchir des lois empiriques associées à la longueur de mélange. Il se compose de l'équation de transport de l'énergie cinétique de la turbulence et d'une équation de transport du taux de dissipation, tirées des équations de Navier Stokes. C'est le modèle $k-\varepsilon$.

\subsection{Prise en compte de la turbulence}

Les grandeurs physiques décomposées sont les suivantes: $\vec{u}=\vec{u}+\vec{u}^{\prime}, \quad c=\bar{c}+c^{\prime}, \rho_{m}=\bar{\rho}_{m}+\rho_{m}^{\prime}$ et $P=\bar{P}+P^{\prime}$. Les quantités surmontées d'une «barre » sont les quantités moyennes, et les quantités suivies d'un « prime » sont les fluctuations. La turbulence est supposée pleinement développée, homogène et isotrope. Ceci est vrai pour de grands nombres de Reynolds ; c'est le cas des écoulements d'avalanche poudreuse. Il est à noter que dans tous les développements suivants, liés à la décomposition de Reynolds et à l'introduction de la moyenne dans les équations, les termes contenant la fluctuation de densité sont largement négligeables devant les autres quantités. Ils sont donc omis.

En introduisant la décomposition de Reynolds dans l'équation (1) et en appliquant l'opérateur moyenne, on obtient l'équation suivante :

$$
\frac{\partial \bar{\rho}_{m}}{\partial t}+\operatorname{div}\left(\bar{\rho}_{m} \vec{u}\right)=0
$$

La prise en compte de la turbulence dans l'équation de conservation de la masse de la neige s'écrit:

$$
\frac{\partial \bar{c}}{\partial t}+\operatorname{div}(\overrightarrow{c \vec{u}})=-\frac{\partial \vec{c} w_{g}}{\partial z}-\operatorname{div}\left\langle c^{\prime} u\right\rangle
$$

L'équation de conservation de la quantité de mouvement est traitée de la même façon:

$$
\frac{\partial \bar{\rho}_{m} \vec{u}}{\partial t}+\operatorname{div} \overline{\vec{F}}+\overrightarrow{\operatorname{grad}}(\bar{P})=\bar{c} \rho_{s} g-\operatorname{div}\langle\vec{F}\rangle
$$

où

$$
\overline{\vec{F}}=\bar{\rho}_{m}[\vec{u} \vec{u} \bar{v} \vec{u} \bar{w} \vec{u}]^{t} \quad \text { et } \quad \bar{F}^{\prime}=\bar{\rho}_{m}\left[u^{\prime} u^{\prime} v^{\prime} u^{\prime} w^{\prime} \vec{u}\right]^{t}
$$


Du fait de la non-linéarité des équations, des termes supplémentaires apparaissent. Ces termes, corrélations d'ordre 2, représentent des flux turbulents de concentration ou de quantité de mouvement au sein de l'écoulement. Les termes de diffusion moléculaire sont très faibles devant les termes de diffusion turbulente et de ce fait sont négligés.

Il reste maintenant à modéliser les termes de corrélation d'ordre 2 qui apparaissent dans ces équations d'évolution :

$$
-\operatorname{div}\langle\vec{F}\rangle \text { et }-\operatorname{div}\left\langle c^{\prime} \vec{u}\right\rangle
$$

La modélisation du flux turbulent de la concentration et de la quantité de mouvement s'appuie sur le modèle de Boussinesq. La viscosité apparente est utilisée sous la forme :

$$
\begin{gathered}
-\operatorname{div}\left\langle c^{\prime} \vec{u}\right\rangle=\operatorname{div}\left(v_{c} \overrightarrow{\operatorname{grad}} \bar{c}\right) \\
-\operatorname{div}\langle\vec{F}\rangle=\operatorname{div}\left(2 \bar{\rho}_{m} v_{c} D-\frac{2}{3} k \bar{\rho}_{m} I\right)
\end{gathered}
$$

où $k$ est l'énergie cinétique de la turbulence :

$$
k=\frac{\left\langle u_{i}^{\prime} u_{i}^{\prime}\right\rangle}{2}, \quad D=\frac{1}{2}\left[\vec{\nabla} \vec{u}+(\vec{\nabla} \vec{u})^{t}\right]
$$

est le tenseur moyen des taux de déformation et $I$ est le tenseur unité.

Finalement, les équations moyennes régissant l'évolution des quantités moyennes intervenant dans l'écoulement, sont les suivantes :

$$
\frac{\partial \bar{\rho}_{m}}{\partial t}+\operatorname{div}\left(\bar{\rho}_{m} \vec{u}\right)=0
$$

$$
\frac{\partial \bar{c}}{\partial t}+\operatorname{div}(\overrightarrow{c \vec{u}})=\operatorname{div}\left(v_{c} \overrightarrow{\operatorname{grad}} \bar{c}\right)-\frac{\partial \vec{c} w_{g}}{\partial z}
$$

$$
\begin{aligned}
& \frac{\partial \bar{\rho}_{m} \vec{u}}{\partial t}+\operatorname{div} \overrightarrow{\vec{F}}+\overrightarrow{\operatorname{grad}}(\bar{P})= \\
& =\left(\rho_{g}^{0}-\bar{\rho}_{g}\right) g+\bar{c} \rho_{s} g+\operatorname{div}\left(2 \bar{\rho}_{m} v_{t} D-\frac{2}{3} k \bar{\rho}_{m} I\right)
\end{aligned}
$$

On suppose que le nombre de Schmidt est de l'ordre de 1. Cela signifie que l'on considère que la diffusion turbulente de la concentration se fait au même taux de diffusion que la quantité de mouvement. En effet la concentration diffuse par l'intermédiaire du fluide; son taux de diffusion est donc équivalent au taux de diffusion de la quantité de mouvement : $v_{c}=v_{t}$.

Il reste donc à déterminer la viscosité turbulente $v$, pour obtenir la fermeture du problème. Pour cela, on adopte le modèle à une équation de la turbulence : cette équation est l'équation d'évolution de l'énergie cinétique de la turbulence. On y adjoint une longueur de mélange empirique qui sera définie ultérieurement.

\subsection{Description du modèle à une équation}

L'énergie cinétique de la turbulence est calculée à partir de l'équation de transport, directement déduite des équations de Navier Stokes. L'équation exacte ainsi établie contient des corrélations inconnues. La modélisation de ces corré- lations, basée sur les mécanismes de " cascades d'énergie » de Kolmogorov, permet alors la fermeture du problème. II faut naturellement dans ce cas, être en présence d'écoulements franchement turbulents, c'est-à-dire à grand nombre de Reynolds. Le coefficient de diffusion turbulente se calcule alors à partir de l'énergie cinétique turbulente, la valeur de celle-ci étant déterminée par l'équation d'évolution. Cette équation d'évolution est obtenue à partir de l'équation de conservation de la quantité de mouvement dans laquelle la décomposition de Reynolds est introduite :

$$
\begin{aligned}
& \frac{\partial k}{\partial t}+\bar{u}_{j} \frac{\partial k}{\partial x_{j}}=-\left\langle u_{i}^{\prime} u_{j}^{\prime}\right\rangle \frac{\partial \bar{u}_{i}}{\partial x_{j}}-2 v\left\langle d_{i j}^{\prime} d_{i j}^{\prime}\right\rangle+ \\
& \mathrm{P}_{\mathrm{r}} \varepsilon \\
&+\frac{\partial}{x_{j}}\left(-\frac{1}{\rho_{m}}\left\langle u_{i}^{\prime} P^{\prime}\right\rangle-\left\langle k u_{j}^{\prime}\right\rangle+2 v\left\langle u_{i}^{\prime} d_{i j}^{\prime}\right\rangle\right) \\
& \mathrm{D}_{\mathrm{iff}}
\end{aligned}
$$

Cette équation fait intervenir plusieurs corrélations multiples, nouvelles inconnues à exprimer et qui font ellesmêmes apparaître de nouvelles corrélations. On se limite alors à l'emploi d'équations pour les corrélations d'ordre 2 , et l'on cherche à exprimer les corrélations inconnues : c'est la fermeture au second ordre. Cette équation d'évolution de l'énergie cinétique de la turbulence est une équation d'advection-diffusion à laquelle s'ajoutent des termes de production et de dissipation de l'énergie cinétique turbulente. Pour modéliser ces termes on se place dans l'hypothèse d'une turbulence pleinement développée vérifiant l'hypothèse de Kolmogorov. Ce qui permet d'écrire :

Dissipation

$$
\varepsilon=v\left\langle\frac{\partial u_{i}^{\prime}}{\partial x_{j}} \frac{\partial u_{i}^{\prime}}{\partial x_{j}}\right\rangle+O\left(\frac{1}{\operatorname{Re}}\right)
$$

Production

$$
\mathrm{P}_{\mathrm{r}}=\left[v_{t}\left(\frac{\partial \bar{u}_{i}}{\partial x_{j}}+\frac{\partial \bar{u}_{j}}{\partial x_{i}}\right)-\frac{2}{3} k \delta_{i j}\right] \frac{\partial \bar{u}_{i}}{\partial x_{j}}
$$

Diffusion

$$
\mathrm{D}_{\text {iff }}=v_{t} \frac{\partial k}{\partial x_{j}}
$$

Le taux de production d'énergie des grosses structures est égal au taux de dissipation de l'énergie des petites structures. Alors : $\varepsilon=C_{\varepsilon} \frac{k^{3 / 2}}{L}$ où $C_{\varepsilon}=0,078, L$ est analogue à une longueur de mélange. $L$ peut être considérée comme l'échelle de longueur caractéristique des grosses structures énergétiques de l'écoulement. L'équation d'évolution de $k$ s'écrit alors sous sa forme définitive :

$$
\begin{array}{r}
\frac{\partial k}{\partial t}+\bar{u}_{j} \frac{\partial k}{\partial x_{j}}=\frac{\partial}{\partial x_{j}}\left(v_{t} \frac{\partial k}{\partial x_{j}}\right)-C_{\varepsilon} \frac{k^{3 / 2}}{L}+\frac{\partial \bar{u}_{i}}{\partial x_{j}}\left[v_{t}\left(\frac{\partial \bar{u}_{i}}{\partial x_{j}}+\frac{\partial \bar{u}_{j}}{\partial x_{i}}\right)\right. \\
\left.-\frac{2}{3} k \delta i j\right]
\end{array}
$$

La viscosité turbulente est reliée à l'énergie cinétique de la turbulence par la formule suivante:

$$
v_{t}=C_{1} L_{k}^{1 / 2} \text { avec } C_{1}=0,43
$$




\section{RÉSOLUTION NUMÉRIQUE DU SYS- TÈME D'ÉQUATIONS}

\subsection{La structure des données}

Le domaine de calcul est discrétisé par des parallélépipèdes de dimensions variables (maillage éléments finis). Les informations concernant chaque élément constituent la structure des données.

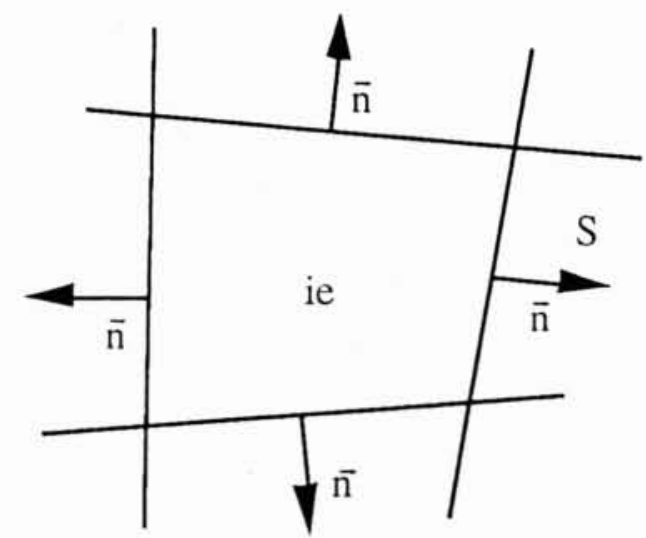

1. Description de la structure de données :

\section{$S$ : surface de l'élément}

$V:$ volume de l'élément

$U_{i e}^{n}$ : valeur au centre de l'élément $i e$ au temps $n$

$U_{i e v e}^{n}$ : valeur au centre de l'élément voisin de l'élément $i e$ au temps $n$.

\subsection{Transformation du système d'équations}

En bidimensionnel, le système des cinq équations à cinq inconnues peut s'écrire sous une forme vectorielle regroupant les termes de transport et de diffusion si l'on omet les barres de «moyennes» et les indices « $m$ » :

$$
\begin{array}{r}
\frac{\partial}{\partial t}\left[\begin{array}{c}
\rho \\
\rho u \\
\rho v \\
\rho w \\
c \\
k
\end{array}\right]+\operatorname{div}\left[\begin{array}{ccc}
\rho u & \rho v & \rho w \\
\rho u^{2}+p & \rho u v & \rho u w \\
\rho u v & \rho v^{2}+p & \rho v w \\
\rho u w & \rho v w & \rho w^{2}+p \\
c u & c v & c w \\
k u & k v & k w
\end{array}\right]= \\
=\operatorname{div}\left(v_{t} \vec{\nabla}\left[\begin{array}{c}
0 \\
\rho u \\
\rho v \\
\rho w \\
c \\
k
\end{array}\right]\right)+\left[\begin{array}{c}
0 \\
0 \\
0 \\
-\rho g \\
0 \\
\mathrm{P}_{\mathrm{r}}-\varepsilon
\end{array}\right]
\end{array}
$$

Cette relation vectorielle s'écrit plus facilement sous la forme condensée suivante :

$$
\frac{\partial U}{\partial t}+\operatorname{div}[F(U)]=\operatorname{div}\left[v_{t} \vec{\nabla} H(U)\right]+G(U)
$$

\subsection{Discrétisation et résolution numérique}

Le système d'équations a été mis sous forme conservative en vue de sa résolution numérique. Le schéma est réalisé en volumes finis, ce qui permet une adaptation aisée à un domaine de forme quelconque (relief d'un couloir d'avalanche), d'ordre deux en espace et un en temps. Dans ce qui suit, après avoir présenté les caractéristiques du maillage de type éléments finis, et la notion de projection, le système est intégré sur la surface de chaque élément entre deux temps consécutifs. L'intégrale de surface est alors transformée en une intégrale sur le pourtour de l'élément. La relation ainsi obtenue est discrétisée dans le temps et l'espace.

A chaque pas de temps, l'étape de projection consiste à affecter à chaque élément du maillage la valeur moyenne du vecteur $U$, obtenue en intégrant $U$ sur l'élément : $U_{i e}^{n}=\frac{1}{S} \int_{S} U \mathrm{~d} S$.

On transforme le système d'équations décrit ci-dessus, en intégrant sur l'élément de maillage ie entre les temps $t(n)$ et $t(n+1):$

$$
\begin{array}{r}
S\left(U_{i e}^{n+1}-U_{i e}^{n}\right)+\int_{t(n)}^{t(n+1)} \int_{S}\left[\operatorname{div} F(U)-\operatorname{div}\left(v_{t} \bar{\nabla}(U)\right)\right. \\
-G(U)] \mathrm{d} S \mathrm{~d} t=0(20)
\end{array}
$$

Le théorème d'Ostrogradsky permet de passer de l'intégrale de volume à l'intégrale de surface:

$$
\begin{aligned}
U_{i e}^{n+1}=U_{i e}^{n}-\frac{\Delta t}{S} \int_{\Gamma}\left[F\left(U^{n}\right) \bar{n}-v_{t} \bar{\nabla}\left(U^{n}\right) \bar{n}\right] \mathrm{d} S & \\
& +\Delta t G\left(U_{i e}^{n}\right)
\end{aligned}
$$

L'intégration se transforme en sommation sur les facettes de l'élément: (if: indice de la facette)

$$
\begin{aligned}
U_{i e}^{n+1}=U_{i e}^{n}- & \frac{\Delta t}{V} \sum_{i f=1}^{4}\left[F\left(U_{i f}^{n}\right) \bar{n}_{i f}\right] S_{i a}+ \\
& +\frac{\Delta t}{V} \sum_{i a=1}^{4}\left[v_{t} \bar{\nabla}\left(U_{i f}^{n}\right) \bar{n}_{i j}\right] S_{i f}+\Delta t G\left(U_{i e}^{n}\right)
\end{aligned}
$$

\subsection{Schéma du second ordre - estimation des gradients}

On construit, à partir des valeurs moyennes, les gradients de $U$ dans le but d'obtenir une approximation de $U$ au second ordre. Les trois composantes du gradient sont obtenues par minimisation de la fonctionnelle :

$$
\begin{aligned}
\psi=\sum_{\text {iev }=K(\text { ie })} & {\left[U_{\text {ieve }}^{n}-\left(U_{\text {ie }}^{n}+\frac{\partial U}{\partial x}\left(x_{\text {ieve }}-x_{c}\right)+\right.\right.} \\
+ & \left.\left.\frac{\partial U}{\partial y}\left(y_{\text {ieve }}-y_{c}\right)+\frac{\partial U}{\partial z}\left(z_{\text {ieve }}-z_{c}\right)\right)\right]^{2}
\end{aligned}
$$

où $K(i e)$ est l'ensemble des éléments voisins de l'élément ie. Dans le calcul des termes de diffusion la valeur du gradient utilisé est le résultat de cette minimisation.

Par contre pour la résolution de la partie hyperbolique du système, il est nécessaire pour préserver la stabilité du schéma, de limiter le gradient de $U$ sur chaque élément afin de ne pas créer de nouveaux extremums locaux. Cette correction est réalisée localement, en calculant les valeurs de $U$ au milieu de chaque facette de ie et en limitant le 
module du gradient de $U$ sur l'élément $i e$ de façon à ce que la valeur sur la facette reste dans l'intervalle engendré par les valeurs moyennes aux centres de mailles des éléments voisins de la facette.

\subsection{Solveur approché - partie hyperbolique}

Pour obtenir la contribution de la partie hyperbolique du système au terme du flux au travers de chaque facette de l'élément, on procède de la façon suivante :

- grâce à la propriété d'invariance par rotation du système d'équation, on écrit le système dans le repère local formé par le plan tangent et la normale à la facette. Ensuite pour réduire le calcul du flux à un cas unidimensionnel on suppose que la variation dans la direction de la tangente est négligeable,

- le problème devenant unidimensionnel, on peut alors utiliser des solveurs approchés du problème de Riemann, posé au niveau de chaque interface, qui peut se schématiser de la façon suivante:

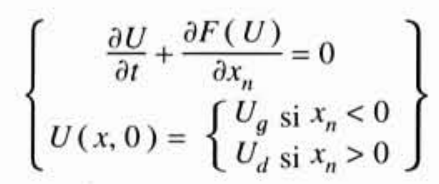

pour chaque interface :

$$
\left\{\begin{array}{l}
U_{g}=U_{i e g .}+(\vec{\nabla} U)_{i e g .}\left(\vec{x}_{\text {interface }}-\vec{x}_{i e g}\right) \\
U_{d}=U_{i e d .}+(\vec{\nabla} U)_{i e d .}\left(\vec{x}_{\text {interface }}-\vec{x}_{i e d}\right)
\end{array}\right\}
$$

où ieg est l'élément à gauche de la facette et ied est l'élément à droite.

La résolution du problème de Riemann permet de déterminer le flux numérique au niveau de l'interface. Il est important de signaler ici la limitation du pas de temps introduite par l'utilisation de ce schéma explicite.

\subsection{Détermination du terme de diffusion}

Le gradient moyen au niveau de chaque interface est estimé par interpolation entre les gradients des éléments voisins de la facette. La diffusion totale au niveau d'un élément est alors déterminée en sommant les flux relatifs à chaque facette.

\subsection{Détermination du modèle d'érosion/dépôt}

Au niveau du contact du sol (substratum) l'écoulement va prélever ou déposer du matériau. Le modèle d'érosion ou du dépôt est fonction de l'intensité turbulente de l'écoulement et de sa concentration ainsi que des caractéristiques du matériau en place. Ainsi quand la contrainte limite de mise en mouvement du matériau est dépassée, les particules sont arrachées par l'écoulement. Par contre quand la vitesse de frottement turbulente est plus faible que la vitesse de chute, le matériau est déposé (sédimentation).

Dans le cas d'un écoulement turbidique permanent établi sur un plan infini, à turbulence homogène l'équation de la conservation de la masse solide s'écrit :

$$
\frac{\partial}{\partial z}\left(w_{f} c\right)+\frac{\partial}{\partial z}\left(v_{t} \frac{\partial c}{\partial z}\right)=0
$$

La solution de cette équation différentielle est :

$$
c=c_{0} e^{\frac{w_{f}}{v_{t}} z}
$$

où $c_{0}$ est la concentration au niveau du sol qui dépend des caractéristiques du matériau et de l'écoulement $c_{0}=f\left(u_{*}, u_{*_{s}}\right) \quad\left(u_{*}\right.$ est la vitesse de frottement locale et $u_{*_{s}}$ correspond à la contrainte limite de mise en mouvement du matériau immobile). L'effet de l'avalanche sur une surface enneigée se fait par l'intermédiaire de la contrainte exercée au niveau du sol. Cette contrainte est $\tau_{f}=\rho_{m} u_{*}^{2}$. En fonction de cette contrainte et en fonction de la contrainte limite d'érosion $\tau_{s}=\rho u_{* s}^{2}$, le flux de masse prélevée ou déposée par l'écoulement se fait de la façon suivante :

- dans le cas d'un excès de contrainte :

$\psi=-u_{*}\left(c-c_{0}\right)$

- et dans le cas d'un déficit de contrainte :

$\psi=-c w_{f}$

\section{IV — VALIDATION DE LA MODÉLISATION}

\subsection{Modélisation physique des courants de turbidité}

Pour appréhender les phénomènes complexes des courants de gravité, des modèles physiques ont été élaborés et développés en laboratoire (modélisation en canal noyé). Leurs buts : déterminer les lois régissant la dynamique de ces écoulements sur surface régulière d'inclinaison constante ou variable, avec présence ou non d'obstacles, ainsi que les variations de la vitesse du front et l'évolution des dimensions de l'aérosol. En effet, les études des phénomènes réels étant difficiles à mettre en œuvre, on a préféré réaliser des études sur modèles réduits qui permettent une souplesse dans les expériences que n'apportent pas les essais réels. Cependant les expérimentations in situ restent indispensables pour valider les modèles physiques et numériques.

Les recherches sur les courants gravitaires ont été progressives, restant souvent fortement liées aux phénomènes des avalanches poudreuses. On peut citer les travaux de Tochon-Danguy et Hopfinger, de Beghin, Hopfinger et Britter pour la modélisation 2D et les travaux de Beghin et Olagne pour la modélisation en 3D.

\subsection{La modélisation physique 2D des courants gravitaires}

On s'intéressera donc à vérifier la validité du modèle par rapport aux expériences réalisées en canal noyé $2 \mathrm{D}$, sur les courants de gravité de type bouffée. Cela consiste dans le modèle physique à étudier l'écoulement produit par un volume fini de fluide dense contenu dans un réservoir que l'on ouvre de façon quasi instantanée dans le canal contenant le fluide ambiant.

On dispose de nombreux essais expérimentaux : $\left(0<\alpha<90^{\circ}\right)$, capacité d'injection et masse volumique variables. Les suspensions de sable permettent de tenir compte des effets de sédimentation. Les parois en Plexiglas des canaux permettent de suivre l'évolution du front de la 
bouffée. Pour estimer les évolutions des paramètres que l'on veut mesurer, le fluide dense est généralement coloré, afin de le distinguer du fluide ambiant. La position du front et les dimensions de la bouffée (longueur et hauteur) sont repérées grâce à un quadrillage tracé sur les parois.

\subsection{Comparaison du modèle et des expériences en canal noyé}

Plusieurs essais ont été utilisés pour tester la validité du modèle numérique ainsi construit. Nous avons utilisé les mesures expérimentales obtenues par Pierre Beghin dans le cas des bouffées de densité bidimensionnelles. Les essais dont les résultats sont présentés ci-dessus sont définis par :

\begin{tabular}{|c|c|c|c|}
\hline Essais & angle $\left({ }^{\circ}\right)$ & $\mathrm{h}(\mathrm{cm}){ }^{*} \mathrm{~L}(\mathrm{~cm})$ & $\mathrm{C}(\%)$ \\
\hline 1 & 20 & 230 & 2 \\
2 & 45 & 80 & 15 \\
3 & 60 & 80 & 15 \\
\hline
\end{tabular}

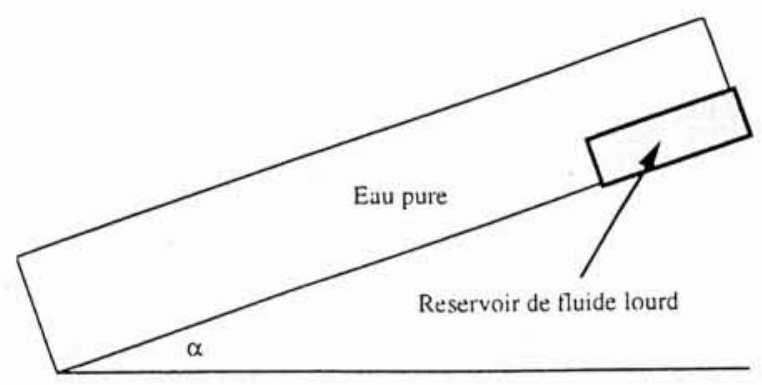

2. Schéma du canal utilisé pour la modélisation des avalanches aérosols 2D.

Il est intéressant d'examiner la partie amont des courbes de vitesses. La vitesse du front de la bouffée subit à l'ouverture du réservoir une accélération assez forte et atteint rapidement son maximum. Dès cet instant, la vitesse décroît rapidement. On peut expliquer cela en remarquant que la turbulence est d'autant plus intense que le mouvement est rapide. Dès l'ouverture de la vanne, la différence de concentration entre les deux milieux est forte. Le fluide lourd est propulsé dans le canal, la vitesse et donc la turbulence augmentent rapidement. Cela provoque un entraînement d'air ambiant important à l'intérieur de la bouffée. Ceci réduit d'autant la différence de concentration entre les milieux. La concentration étant moins importante, la vitesse de la bouffée diminue.

D'une manière générale, dans la partie amont du canal, l'estimation de la vitesse du front issue des résultats numériques est toujours supérieure ou égale aux valeurs expérimentales. Par contre, dans la partie amont, cette vitesse est généralement mieux prédite par le modèle.

Pour la phase établie ainsi que pour la phase de décélération, nous disposons de mesures qui montrent que les résultats du modèle sont globalement assez proches des résultats expérimentaux. Pour la phase d'accélération, sa dynamique est surtout montrée par les simulations numériques. En effet nous ne disposons pas de mesures expérimentales dans la phase d'accélération.

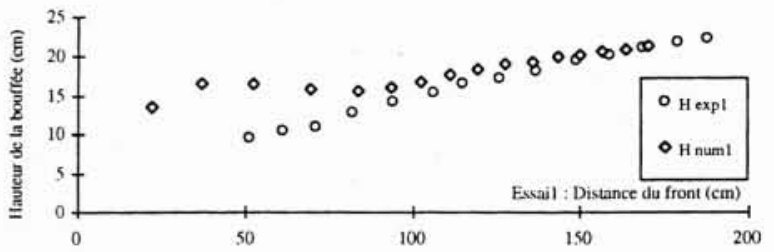

3. Hauteur du front en fonction de la distance parcourue.

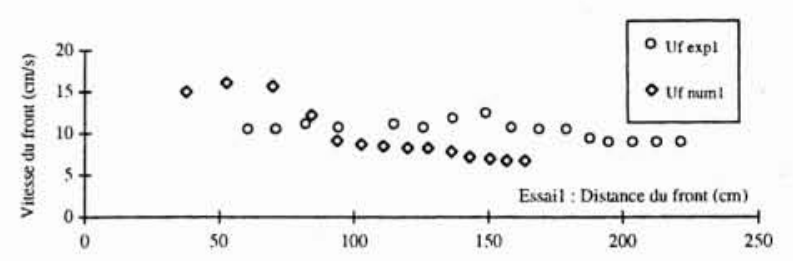

4. Vitesse du front en fonction de la distance parcourue.

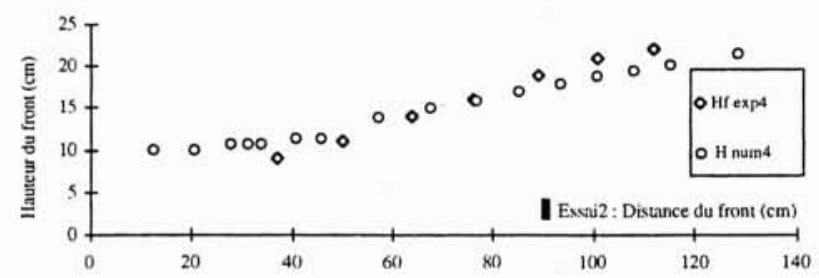

5. Hauteur du front en fonction de la distance parcourue.

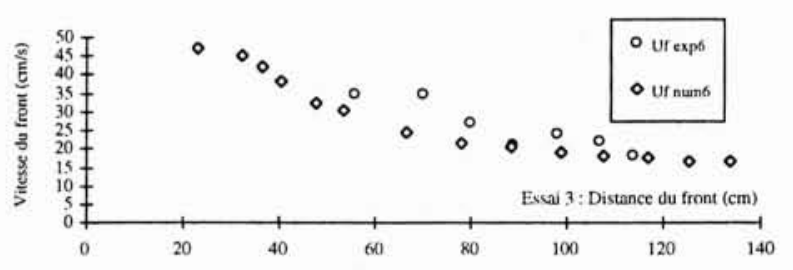

6. Vitesse du front en fonction de la distance parcourue.

\section{$\mathrm{V} \square \mathrm{CONCLUSION}$}

L'étude qualitative des résultats numériques montre que le modèle prédit correctement les évolutions des hauteurs et des vitesses de front de la bouffée bidimensionnelle. Les caractéristiques des avalanches aérosols, et plus généralement des courants gravitaires se retrouvent dans les résultats numériques: centre de vorticité, entraînement d'air, tête de la bouffée surélevée, traînée arrière.

Des comparaisons quantitatives entre l'expérience et le modèle numérique, nous pouvons dégager les conclusions suivantes :

- les expériences réalisées avec des solutions salines sont peu nombreuses, les résultats étant parfois peu évidents à exploiter et surtout uniques ;

- la comparaison des résultats entre le modèle numérique de type gazeux et le modèle expérimental de type aqueux n'est pas forcément bien adaptée ; 
- les vitesses du front de la bouffée sont trop fortes dans la partie amont du canal et trop faibles dans la partie aval. Cela peut provenir d'une dissipation trop importante, la turbulence étant surestimée par le modèle. Il est à signaler que pour des nombres de Reynolds faibles, l'écoulement des suspensions n'est plus turbulent ;

- les hauteurs sont globalement assez bien reproduites. Cependant, les hauteurs estimées en fin de simulation sont toujours plus faibles que celles obtenues expérimentalement. Là aussi, il semble probable que la modélisation de la turbulence est à remettre en cause.

\section{Bibliographie}

[1] Beghin P., Olagne X. (1991). - Experimental and theorical study of the dynamics of powder snow avalanches. Cold Regions Science and Technology, vol. 19.

[2] BEGHIN P. (1979). - Etude des bouffées bidimensionnelles de densité en écoulement sur pente avec application aux avalanches de neige poudreuse. Grenoble : Université Joseph Fourier, (Thèse de doctorat).
[3] Brandstatter W., Wieser K., Schaffhauser H. - Three dimensional simulation of powder avalanches.

[4] Cousteix J. (1989). - Turbulence et couche limite. Cepadues Edition.

[5] Hopfinger E.J., TOCHON-DANGUY J.C. (1977). - A model study of powder snow avalanche. Journal of Glaciology, vol. 19, pp. 343-356.

[6] NAAIM M. - Free surface flow modelling on a complex topography. Proceeding of the speciality conference: Modelling of flood propagation over initially dry areas, Asce, Milan, juin 94

[7] NAAIM M. et MARTINEZ H. - Experimental and theoretical determination of concentration profiles and influence of particle characteristics in blowing snow, à paraitre dans Surveys in Geophysics.

[8] SIMPSON J.E. (1987), - Gravity currents in the environment and the laboratory. Ellis Horwood series in Environmental Science. 